\title{
Metabolic effects of air pollution exposure and reversibility
}

\author{
Sanjay Rajagopalan, ${ }^{1,2}$ Bongsoo Park, ${ }^{3}$ Rengasamy Palanivel, ${ }^{1}$ Vinesh Vinayachandran, ${ }^{1}$ Jeffrey A. Deiuliis, ${ }^{1}$ Roopesh Singh Gangwar, ${ }^{1}$ \\ Lopa Das, ${ }^{1}$ Jinhu Yin, ${ }^{3}$ Youngshim Choi, ${ }^{3}$ Sadeer Al-Kindi, ${ }^{1}$ Mukesh K. Jain, ${ }^{1,2}$ Kasper D. Hansen, ${ }^{4}$ and Shyam Biswal ${ }^{3}$
}

'Cardiovascular Research Institute, Case Western Reserve University, Cleveland, Ohio, USA. ${ }^{2 H a r r i n g t o n ~ H e a r t ~ a n d ~ V a s c u l a r ~ I n s t i t u t e, ~ U n i v e r s i t y ~ H o s p i t a l ~ C l e v e l a n d ~ M e d i c a l ~ C e n t e r, ~ C l e v e l a n d, ~ O h i o, ~ U S A ~}$ ${ }^{3}$ Department of Environmental Health and Engineering and ${ }^{4}$ Department of Biostatistics, Johns Hopkins Bloomberg School of Public Health, Johns Hopkins University, Baltimore, Maryland, USA.

\begin{abstract}
Air pollution involving particulate matter smaller than $2.5 \mu \mathrm{m}$ in size $\left(\mathrm{PM}_{2.5}\right)$ is the world's leading environmental risk factor contributing to mortality through cardiometabolic pathways. In this study, we modeled early life exposure using chow-fed C57BL/6J male mice that were exposed to real-world inhaled, concentrated $\mathrm{PM}_{2.5}\left(\sim 10\right.$ times ambient levels $\left./ \sim 60-120 \mu \mathrm{g} / \mathrm{m}^{3}\right)$ or filtered air over a 14-week period. We investigated the effects of $\mathrm{PM}_{2.5}$ on phenotype, the transcriptome, and chromatin accessibility and compared these with the effects of a prototypical high-fat diet (HFD) as well as cessation of exposure on phenotype reversibility. Exposure to $\mathrm{PM}_{2.5}$ impaired glucose and insulin tolerance and reduced energy expenditure and ${ }^{18}$ FDG-PET uptake in brown adipose tissue. Multiple differentially expressed gene clusters in pathways involving metabolism and circadian rhythm were noted in insulin-responsive tissues. Although the magnitude of transcriptional change detected with $\mathrm{PM}_{2.5}$ exposure was lower than that observed with a HFD, the degree of alteration in chromatin accessibility after $\mathrm{PM}_{2.5}$ exposure was significant. The novel chromatin remodeler SMARCA5 (SWI/SNF complex) was regulated in response to $\mathrm{PM}_{2.5}$ exposure, the cessation of which was associated with a reversal of insulin resistance and restoration of chromatin accessibility and nucleosome positioning near transcription start sites, as well as a reversal of exposure-induced changes in the transcriptome, including SMARCA5. These changes indicate pliable epigenetic control mechanisms following cessation of exposure.
\end{abstract}

\section{Introduction}

Air pollution is the leading environmental cause of premature reversible death and disability in the world today (1). A large body of evidence implicates the component of air pollution containing particulate matter smaller than $2.5 \mu \mathrm{m}\left(\mathrm{PM}_{2.5}\right)$ in size in the development of cardiovascular disease risk factors such as hypertension, insulin resistance (IR), and type 2 diabetes mellitus (T2DM) $(2,3)$. Although the mechanisms by which inhalation of $\mathrm{PM}_{2.5}$ induces IR and T2DM remain unclear, a constellation of responses including inflammation and redox stress have been implicated. To our knowledge, no studies have examined the metabolic, transcriptomic, and epigenetic effects of air pollution in comparison with other stressors such as a high-fat diet (HFD). Importantly, the reversibility of $\mathrm{PM}_{2.5}$-induced transcriptional and epigenetic changes has not, to our knowledge, been examined. In this study, we evaluated the metabolic phenotypic, transcriptional, and epigenomic changes in response to exposure to concentrated ambient $\mathrm{PM}_{2.5}$ (referred to hereafter as $\mathrm{PM}_{2.5}$ ) and the response of these parameters to cessation of exposure.

Authorship note: SR and BP contributed equally as first authors.

Conflict of interest: The authors have declared that no conflict of interest exists. Copyright: (2) 2020, American Society for Clinical Investigation.

Submitted: February 24, 2020; Accepted: July 29, 2020; Published: October 12, 2020.

Reference information: / Clin Invest. 2020;130(11):6034-6040.

https://doi.org/10.1172/JCl137315.

\section{Results and Discussion}

Air pollution exposure. Three-week-old C57BL/6J mice on a chow diet were started on exposure to $\mathrm{PM}_{25}$ for 14 weeks. Figure 1 provides a summary of the systemic effects of ambient air pollution on IR in $\mathrm{PM}_{2.5}$-exposed male mice. $\mathrm{PM}_{2.5}$ was delivered inhalationally ( $10 \times$ ambient level $/ 60-120 \mu \mathrm{g} / \mathrm{m}^{3}$ for 6 hours/day, 5 days/week) using a versatile aerosol concentrator and enrichment system (Supplemental Figure 1 indicates the $\mathrm{PM}_{2.5}$ concentrations during the exposure period [the mean $\mathrm{PM}_{2.5}$ concentration was $80 \mu \mathrm{g} / \mathrm{m}^{3}, \sim 10 \times$ ambient concentration], the meteorological conditions, and the elemental characterization of the air pollution exposure; supplemental material available online with this article; https://doi.org/10.1172/ JCI137315DS1). Three-week-old C57BL/6J mice were fed a HFD for 14 weeks and served as a positive control for IR and associated transcriptomic and epigenomic changes.

$I R$ and metabolic dysfunction with air pollution exposure. $\mathrm{PM}_{2.5}$ exposure resulted in alterations in glucose clearance compared with filtered air (FA) in male mice. Insulin responsiveness (Figure 1, B and C) was also altered by $\mathrm{PM}_{2.5}$ exposure. We observed no difference in mean body weights between the groups (Supplemental Figure 2A). Metabolic cage experiments demonstrated a reduction in $\mathrm{VO}_{2}, \mathrm{VCO}_{2}$, and energy expenditure (EE) in $\mathrm{PM}_{2.5}$-exposed mice during both light (Zeitgeber time 0-12 [ZTO-ZT12]) and dark (ZT12-ZT24) phases (Figure 1, D-F). $\mathrm{PM}_{2.5}$ exposure reduced the respiratory quotient only at nighttime (Supplemental Figure 2D). PM $_{2.5}$-exposed mice had reduced glucose uptake in brown adipose tissue (BAT) as measured by ${ }^{18}$ FDG-PET (Figure 1G). The body mass of both male and 

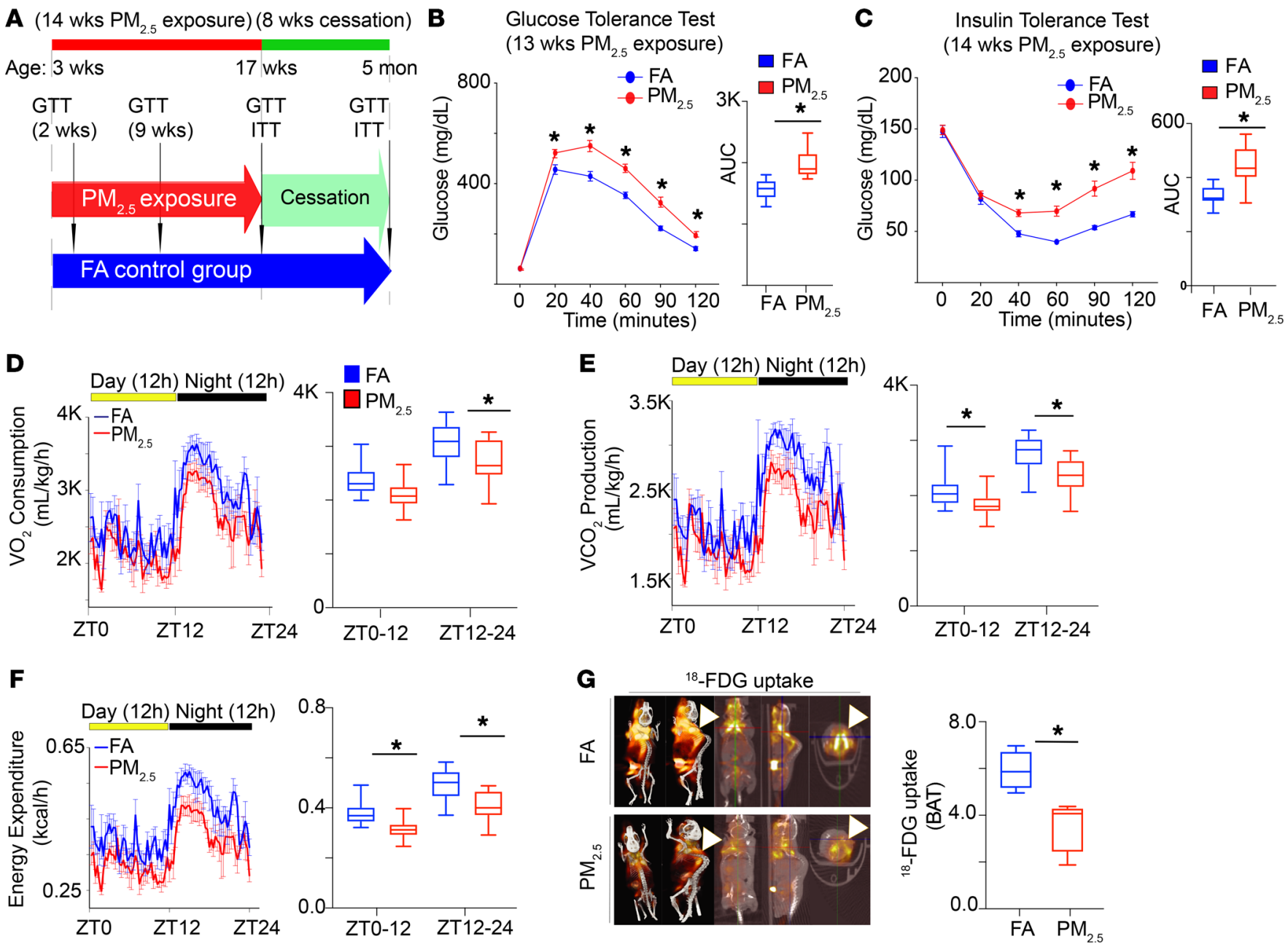

Figure 1. Systemic effects of ambient air pollution $\left(\mathbf{P M}_{2.5}\right)$ on IR. (A) Experimental plan including a cessation period of 8 weeks following 14 weeks of exposure to FA or $\mathrm{PM}_{2.5^{\circ}}$ (B) GTT (intraperitoneal administration of glucose, $2 \mathrm{~g} / \mathrm{kg}$ body weight) and AUC ( $n=12 / \mathrm{group}$ ). (C) ITT (intraperitoneal administration of insulin, $0.75 \mathrm{U} / \mathrm{kg}$ body weight) and $\mathrm{AUC}\left(n=12 /\right.$ group). (D) $\mathrm{VO}_{2}$ consumption, (E) $\mathrm{VCO}_{2}$ production, and (F) EE. Line graphs indicate averages of the day and night cycles over a 48-hour period, and bar graphs indicate the total value at the indicated time points $(n=6)$. (G) Representative PET image (whole-body ${ }^{18} \mathrm{FDG}$ uptake) of FA- and PM 2.5 -exposed mice ( $n=4 /$ group) and quantitative measurement of ${ }^{18} \mathrm{FDG}$ uptake from BAT (value of region of interest [ROI]). Data are expressed as the mean \pm SEM. ${ }^{*} P<0.05$ relative to FA mice, as determined by Student's $t$ test or, as appropriate, 2 -way ANOVA.

female mice increased in response to a HFD, but not to $\mathrm{PM}_{25}$ exposure (Supplemental Figure 2A). $\mathrm{PM}_{25}$ induced abnormalities in glucose clearance and insulin responses that were comparable to those seen with a HFD, but only in males (Supplemental Figure 2, B and C). Given that phenotypic changes were seen only in male mice, we restricted further analysis to males. We acknowledge that it is entirely possible that there could still be conserved responses in males and females that diverge from the sexual dimorphic responses of glucose tolerance and insulin responsiveness. Hepatic cholesterol, but not plasma cholesterol, increased in the $\mathrm{PM}_{2.5}$-exposed group, whereas lipid deposition and fibrosis were increased in both $\mathrm{PM}_{2.5}$ - and HFDexposed groups (Supplemental Figure 3, A and B). Hepatic inflammation in $\mathrm{PM}_{2.5}$-exposed and HFD-fed mice was characterized by an elevation of proinflammatory M1 genes (TNF- $\alpha$, IL-6, and TLR4) and downregulation of antiinflammatory M2 genes (IL-10) (Supplemental Figure 3, C and D). We found that liver adiponectin levels were markedly downregulated in response to $\mathrm{PM}_{2.5}$ exposure. In many of these genes, the degree of regulation and directionality in hepatic tis- sue was comparable to that seen with a HFD (Supplemental Figure 3, $\mathrm{C}$ and D). Hepatic glycogen levels were decreased in $\mathrm{PM}_{25}$-exposed mice, albeit to a lesser degree, compared with levels in HFD-fed mice (Supplemental Figure 3E).

In summary, the phenotype encountered with chronic air pollution exposure was characterized by alterations in oxygen consumption and EE, hepatic inflammation, elevated triglycerides with evidence of hepatic steatosis, and glycogen depletion. Interestingly, mice exposed to air pollution did not develop adiposity, similar to the phenotype of lean IR that is common in many parts of Asia. Importantly, we observed a strong sexually dimorphic response, as only male mice developed IR.

Differential transcriptome and functional annotation of $P M_{2.5}$ versus HFD exposure. We performed RNA-Seq to quantify transcriptomes using RNA from liver, skeletal muscle, BAT, white adipose tissue (WAT), and hypothalamus from FA- and $\mathrm{PM}_{2.5}{ }^{-}$ exposed mice, which resulted in the identification of multiple differentially expressed genes (DEGs) (Figure 2A). Supplemental 
A

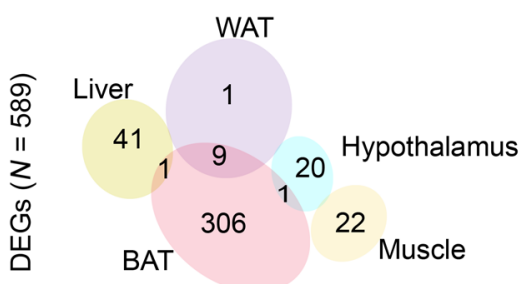

$(\mathrm{FDR}<0.05, \log \mathrm{CPM}>1)$

C

FA vs. $\mathrm{PM}_{2.5}$

Cancer
progression

Enriched Functional Terms

Chow-FA vs. HFD

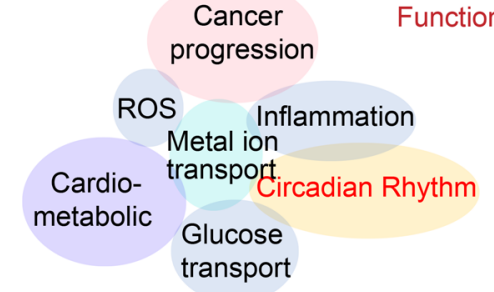

D

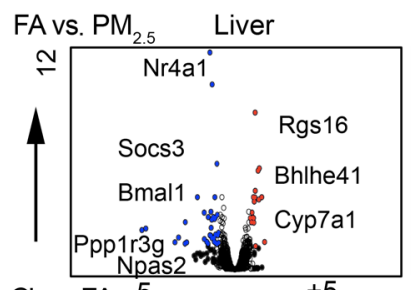

Chow-FA -5

vs. HFD

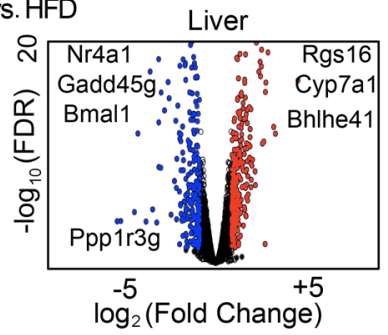

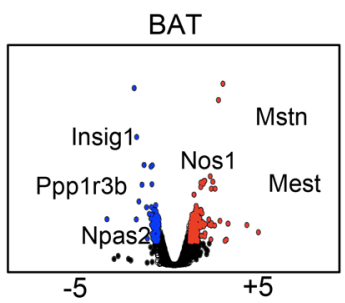

$-5$

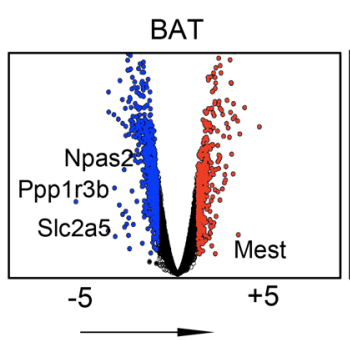

B

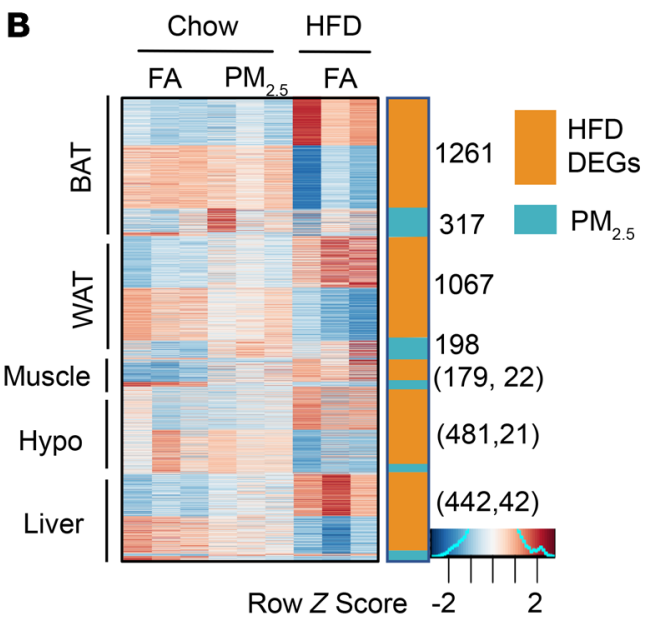

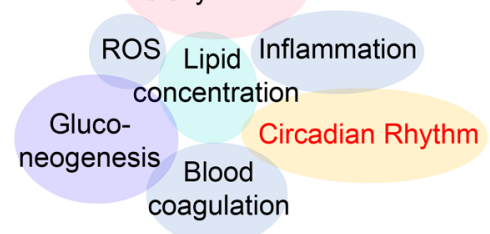

Fatty acid

biosynthesis
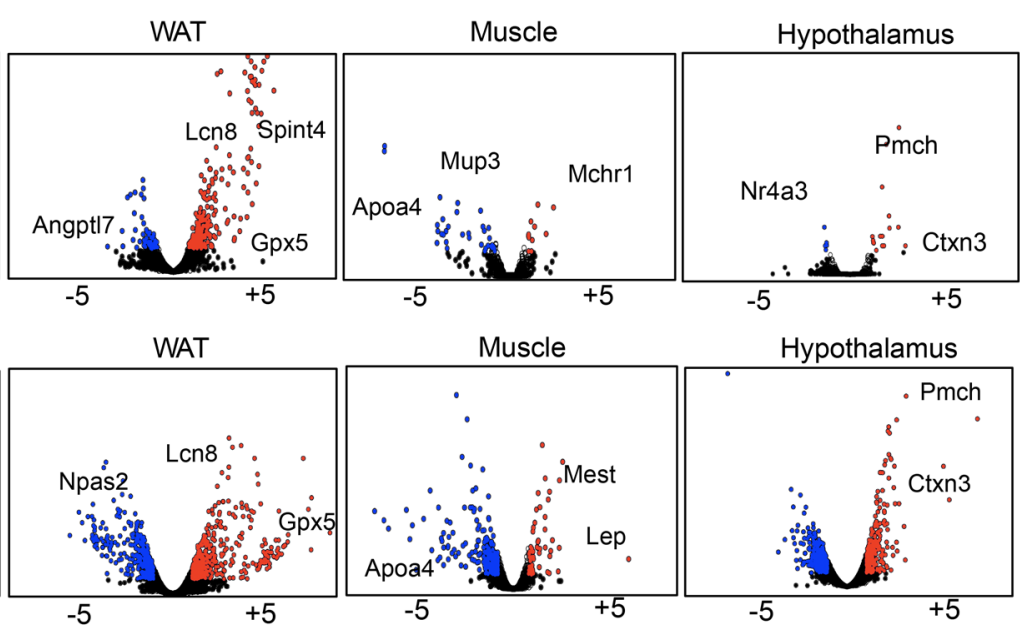

Hypothalamus

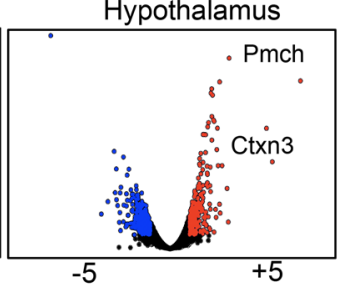

Figure 2. Comprehensive transcriptome analysis in insulin-responsive tissues. (A) Venn diagram depicting DEGs in various tissues from FA- and $\mathrm{PM}_{2.5}$-exposed mice (FDR $<0.05$, logCPM $>1$ ). (B) Tissue-specific DEGs and overlapped genes in FA, PM ${ }_{2.5}$, and HFD-exposed mice $(n=45)$. (C) Summary of enriched $\mathrm{CO}$ terms and functional pathways in FA, PM 2.5 , and HFD transcriptomes. (D) Volcano plots compare a chow diet versus a HFD and FA versus PM 2.5 exposure, indicating selected DEGs of IR and stress response elements in various tissues from HFD-fed mice.

Figure 4, A-F, shows the results of our principal component analysis (PCA) according to the tissue of origin and exposure status. We identified a total of 589 DEGs for $\mathrm{PM}_{2.5}$ exposure versus FA exposure, with 11 genes being differentially expressed in 2 tissue types (Venn diagram, Figure 2A, and hypergeometric test, Supplemental Table 2). The HFD appeared to induce large transcriptional changes across all tissue types (Figure 2B). In contrast, $\mathrm{PM}_{2.5}$ changes were less pronounced, with the largest number of DEGs in BAT, followed by WAT and liver (Figure 2B). The most enriched gene ontology (GO) terms for the $\mathrm{PM}_{2.5}$ transcriptome were related to cancer progression, cardiometabolic function, and circadian rhythm, and the corresponding pathways associated with these were inflammation, redox stress, metal ion transport, and glucose metabolism (Figure 2C). In contrast, a HFD induced changes in fatty acid biosynthesis, inflammation, gluconeogenesis, and lipid regulatory pathways. More detailed DEGs, functional annotations, and potential upstream and downstream regulators are summarized in Supplemental Figures 5-9. Supplemental Figure $4 \mathrm{G}$ depicts the number and directionality of DEGs in response to $\mathrm{PM}_{2.5}$ exposure and a HFD when compared with FA exposure. In liver, WAT, hypothalamus, and muscle, the number of DEGs that were concordant was higher than the number of genes displaying a discordant response. We detected comparable numbers of discordant and concordant DEGs in BAT (Supplemental Figure 4G).

$D E G$ s in insulin-responsive tissue and functional annotations. $\mathrm{PM}_{2.5}$ exposure induced 42 DEGs in the liver, 35 of which overlapped with HFD-induced DEGs (Supplemental Figure 4G). GO analysis revealed that circadian rhythm genes in the liver ranked at the top (GO:0007623 circadian rhythms). These genes included transcriptional regulators (e.g., Dbp, Bhlhe41, Cry1, Per3, and Arntl/Bmal1). Although the core clock components cryptochrome 1 (Cry1) and Bmal1 (Arntl1) were downregulated with $\mathrm{PM}_{2.5}$ expo- 
A $\quad \mathrm{PM}_{2.5} \mathrm{DEG}$

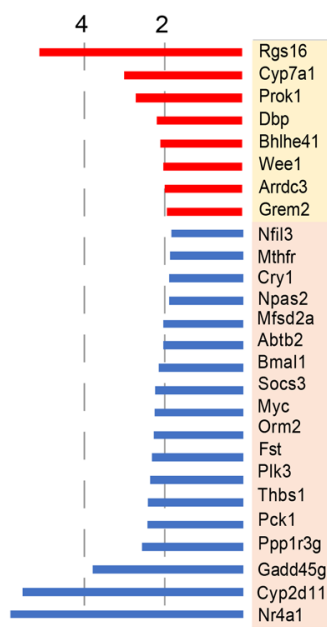

Representative protein coding DEGs

(e.g. circadian genes, inflammatory pathway)
HFD DEG

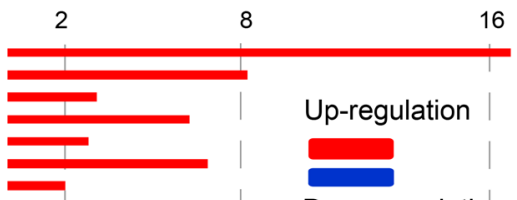

Down-regulation

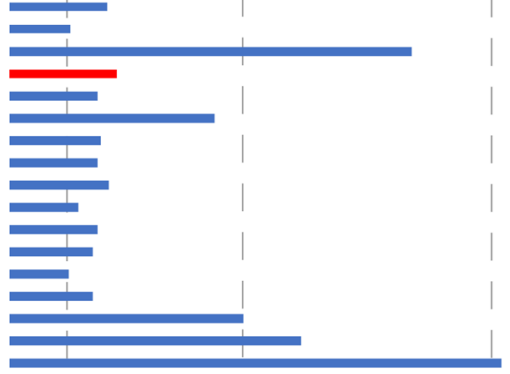

Fold Change

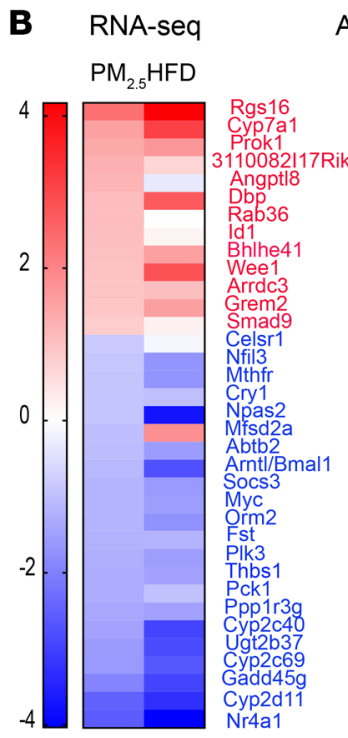

$\log _{2}(\mathrm{FC})$

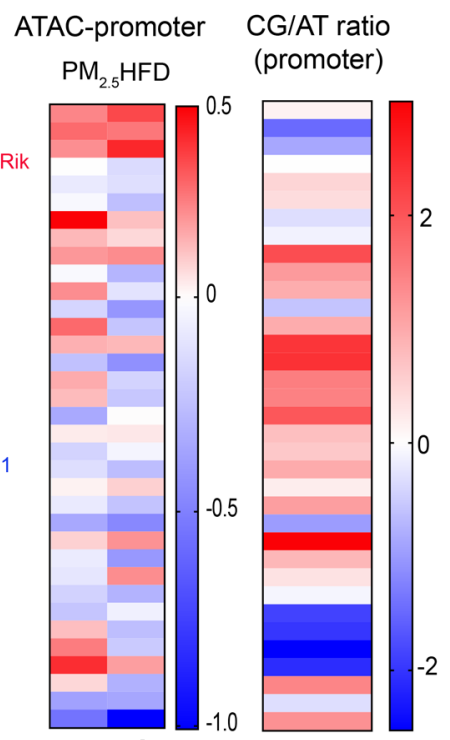

$\log _{2}(\mathrm{FC})$

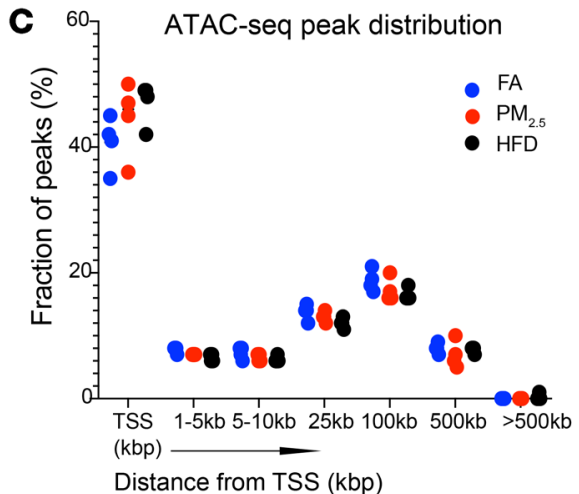

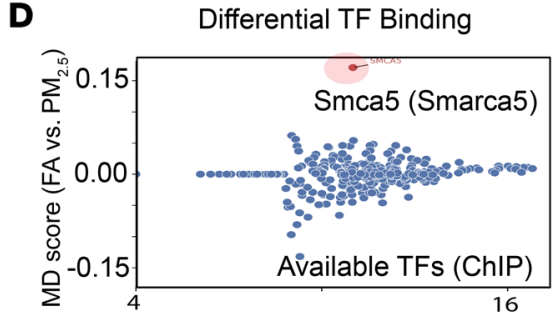

Sum of the no. of peaks overlapping by $3 \mathrm{~kb}$

FA

$P M$

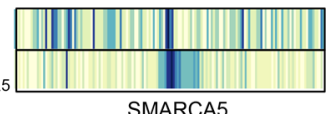

E

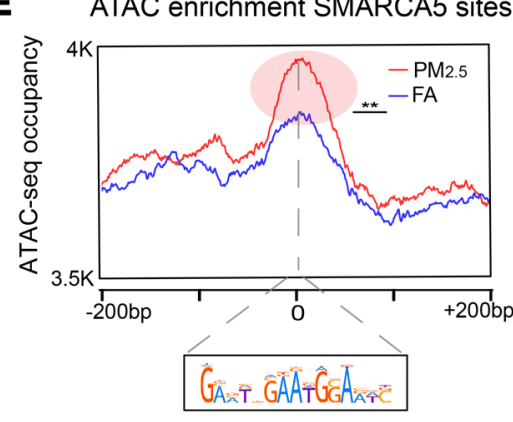

Distance from SMARCA5 motif (bp)

Figure 3. Liver transcriptomic and epigenomic changes. (A) Comparison of the expression levels of DEGs (enriched inflammatory and circadian genes) between $\mathrm{PM}_{2.5}$ - and HFD-exposed mice. (B) Integrative analysis using RNA-Seq and ATAC-Seq data sets and the corresponding CpC content in promoters. FC, fold change. (C) Open chromatin distribution across the 3 groups (FA, $\mathrm{PM}_{2.5}$, and HFD). (D) Differential transcription factor (TF) binding analysis using the available ChIP-Seq database. MD, motif displacement. (E) ATAC-Seq enrichment on the SMARCA5 binding motifs. ${ }^{*} P<0.01$, by Kolmogorov-Smirnov test.

sure, the transcriptional repressor basic helix-loop-helix family member e41 (Bhlhe41) was upregulated (4). Ppp1r3g, a markedly downregulated gene with $\mathrm{PM}_{25}$ exposure, belongs to a family of glycogen-targeting regulatory subunits ( $G$ subunits) that coordinate glycogen synthesis by targeting the catalytic subunit of PP1 to the glycogen particles and that activate glycogen synthase through PP1-mediated dephosphorylation (5). These findings were consistent with glycogen depletion in response to $\mathrm{PM}_{2.5}$ exposure (Supplemental Figure 3D). Several G protein-coupled receptor proteins (C5ar1, Celsr1, Adgrv1) were downregulated in the liver, including the nuclear receptor protein Nr4a1 (Nur77) (Figure 2D). Previous studies have identified Nr4a1 as a transcriptional regulator of glucose utilization in the liver and macrophage polarization $(6,7)$. Rgs16, a regulator of $G$ protein signaling shown to restrict the proinflammatory response of monocytes, was the most upregulated gene in the liver (8). In addition, Supplemental Figure $7 \mathrm{E}$ depicts the TGF- $\beta$ signaling pathway and the corresponding $\mathrm{PM}_{2.5}{ }^{-}$and HFD-specific DEGs. We also observed the downregulation of mTOR signaling DEGs (Supplemental Figure 8A, FDR
$<0.1)$. The enriched functional pathways and diseases for skeletal muscle and hypothalamus are summarized in Supplemental Figure 9. BAT, WAT, and liver tissues had the largest number of overlapping DEGs between $\mathrm{PM}_{2.5}$ - and HFD-exposed mice (Supplemental Figure 4G). In contrast, skeletal muscle and hypothalamus showed a smaller number of common DEGs in response to a HFD and $\mathrm{PM}_{2.5}$. Enriched pathway terms specific for BAT DEGs upregulated specifically with $\mathrm{PM}_{2.5}$ exposure included T2DM signaling and neuronal NOS (nNOS) signaling (Supplemental Figure 5B). We also observed downregulation of adipogenesis DEGs (Ccng2, Acly, Slc25a1, Angptl4) and upregulation of inflammatory response DEGs in gene set enrichment analysis of BAT (Supplemental Figure 5C). Protein phosphatase 1 regulatory subunit 3G (Ppp1r3b) in BAT, an analog of Ppp1r3g, was markedly downregulated with both $\mathrm{PM}_{2.5}$ and HFD exposure (Figure 2D), whereas myostatin (Mstn), known to be linked to diabetes (9), was markedly upregulated in BAT (Figure 2D). Supplemental Figure 6 depicts functional links between the upregulated genes in WAT and their corresponding pathways. 
A

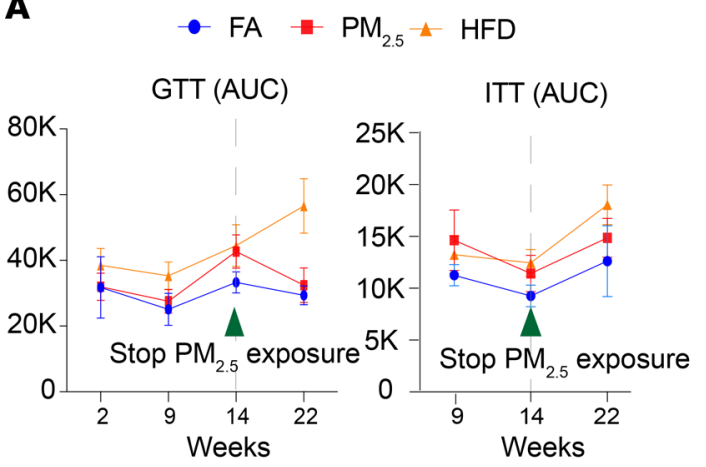

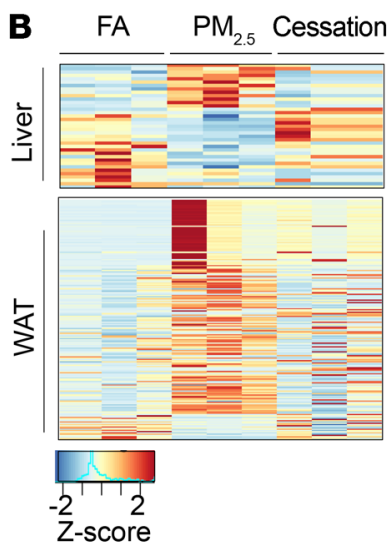

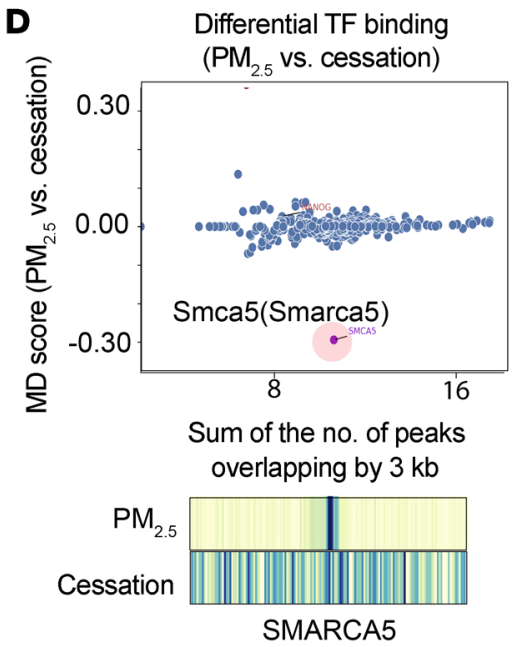

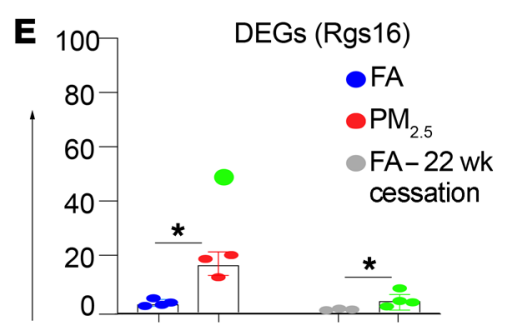

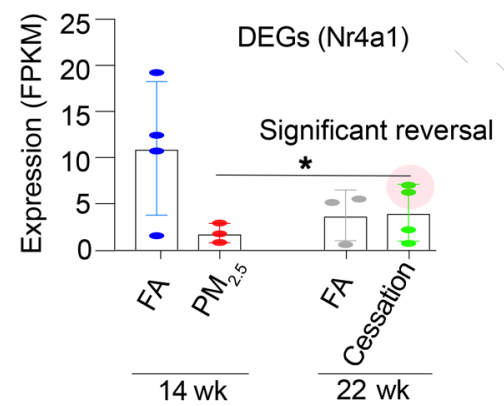

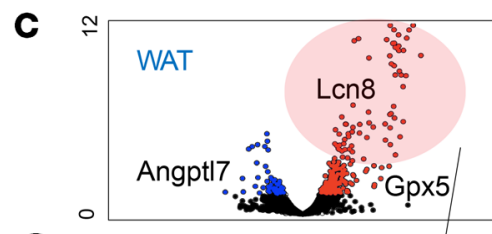
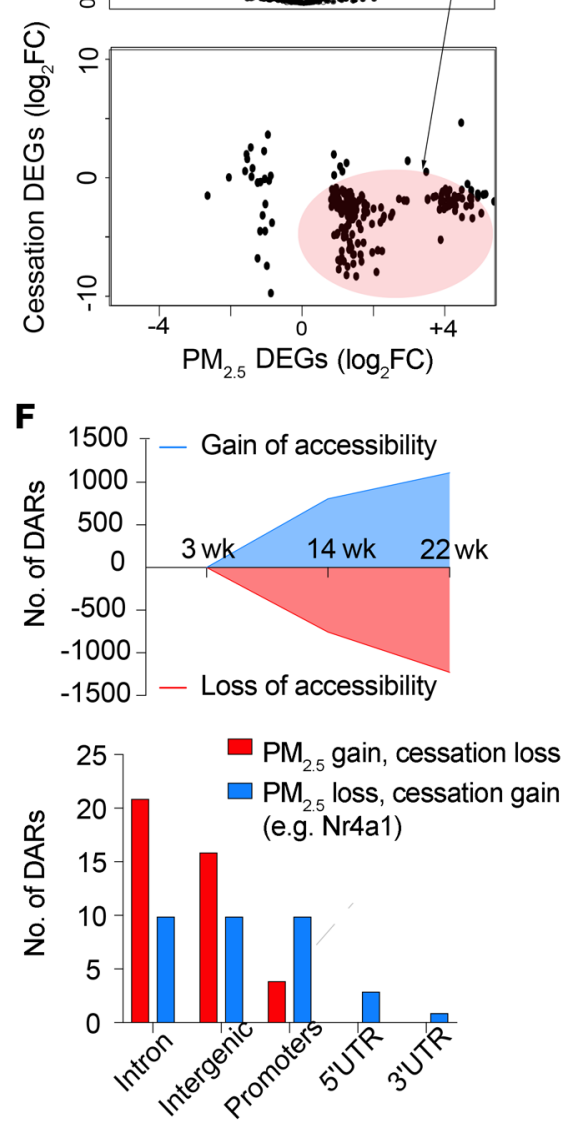

Figure 4. Impact of exposure cessation versus continued PM $_{2.5}$ exposure in liver. (A) Comparison of GTTs and ITTs, as represented by the AUC, in FA-, PM ${ }_{2.5}^{-}$, and HFD-exposed mice at the indicated time points. Dashed line indicates the cessation of exposure at 14 weeks. (B) Heatmap of liver and WAT DEGs by week 8 after $\mathrm{PM}_{2.5}$ exposure cessation (14-22 weeks). (C) Volcano plot of the WAT transcriptome at 14 weeks and scatter plot of the DEC fold change distribution showing a reversal of highly upregulated genes in $\mathrm{PM}_{2.5}$-exposed mice. (D) Differential transcription factor binding analysis using ATAC-Seq data sets and the reversal of SMARCA5 binding profiles ( $\mathrm{PM}_{2.5}$ vs. cessation). (E) Quantitative assessment summarizing normalized transcript values for 2 select genes (Rgs16 and Nr4a1) across mouse samples. FPKM, fragments per kilobase per million mapped reads. (F) Summary of reversible DARs and genomic regions.

Our results support epidemiologic and empirical observations linking air pollution with pathways that promote susceptibility to other noncommunicable diseases (1-3). Circadian rhythm alterations constitute a common denominator for the development of cancer as well as metabolic and cardiovascular diseases (10-12). Many circadian factors are classic epigenetic regulators, and, conversely, many metabolites affect epigenetic regulators and the epigenomic landscape $(13,14)$.

Changes in chromatin accessibility in response to $P M_{2.5}$ exposure and $a$ HFD. Given that $\mathrm{PM}_{2.5}$ exposure resulted in a distinct transcriptomic response (Figure $3 \mathrm{~A}$ ) similar to that elicited by a HFD, we hypothesized that DEGs may be regulated by epigenetic reprogramming driven by differential transcription factor binding (Supplemental Figure 10). Using ATAC-Seq, we evaluated genome-wide chromatin accessibility in the livers of $\mathrm{PM}_{2.5}-$ exposed mice and compared the results with those for HFD-fed mice (Figure $3 \mathrm{~B})$. We analyzed the ATAC-Seq data sets on the basis of fragment size distribution and identified uniquely reproducible peaks in the $\mathrm{PM}_{2.5}$ and HFD groups (Supplemental Figure 11, A and B). We further identified differentially accessible regions (DARs) and found that 218 were common gain-of-accessibility regions and 124 were common loss-of-accessibility regions (Supplemental Figure 11C). We also explored the degree of concordance and discordance between transcriptomic and epigenomic alterations in response to a HFD and $\mathrm{PM}_{2.5}$ exposure (Figure 3, B and C). The DARs generally corresponded with upregulated gene expression and, conversely, less accessible regions with downregulated gene expression. We summarized the CG/AT ratio on the promoter sites (Figure 3B) as a reflection of chromatin accessibility, corresponding to CpG-rich regions. Of the reproducible peaks that overlapped with both FA and $\mathrm{PM}_{2.5}, 1937$ peaks were differentially accessible with $\mathrm{PM}_{2.5}$ exposure (Supplemental Figure 11C). Among those peaks, we searched for $\mathrm{PM}_{2.5}$-specific differential "distal" peaks, denoting potential distal regulatory 
sites (1-5 kb, $10 \mathrm{~kb}, 25 \mathrm{~kb}, 100 \mathrm{~kb}, 500 \mathrm{~kb}$ and >500 kb) (Figure $3 \mathrm{C}$ ), and performed differential motif binding analysis (15) using publicly available ChIP-Seq data sets (16). We found the chromatin remodeler SMARCA5 (SWI/SNF-related matrix-associated actin-dependent regulator of chromatin subfamily A member 5) to be a differential binding protein in $\mathrm{PM}_{2.5}$-exposed mice when compared with FA-exposed mice (Figure 3, D and E).

Epigenetic reprogramming in response to environmental exposure may represent a critical buffer against an adverse health response through the regulation of gene expression and chromosome integrity (17). Circadian rhythm genes in the liver ranked at the top of the GO analysis in our study, with changes noted in core clock components and transcriptional regulators of circadian rhythm. Although the core clock components cryptochrome 1 (Cry1) and Bmal1 (Arntl1) were downregulated in response to $\mathrm{PM}_{2.5}$, we found that the transcriptional repressor of the clockArntl/Bmal1 heterodimer basic helix-loop-helix family member e41 (Bhlhe41) was upregulated.

Cessation of $\mathrm{PM}_{2.5}$ exposure leads to reversal of transcriptomic and epigenomic changes. After 8 weeks of cessation of $\mathrm{PM}_{2.5}$ exposure, we noted an improvement in glucose tolerance and insulin sensitivity (Figure 4A). Given the degree of phenotypic variation in reversibility (Supplemental Figure 12, A and B), we were interested in the corresponding transcriptomic and epigenomic changes. Figure 4B shows the reversal of a number of highly regulated genes in liver and WAT, with a marked reversibility within 8 weeks of exposure cessation (Figure $4 \mathrm{C}$ and Supplemental Figure 13). We performed ATAC-Seq on liver to identify possible chromatin accessibility changes indicating a reversal mechanism after cessation of $\mathrm{PM}_{2.5}$ exposure. Supplemental Figure 14A depicts DARs in response to $\mathrm{PM}_{2.5}$ cessation in liver. In total, we discovered 3467 DARs that changed in the liver following $\mathrm{PM}_{2.5}$ cessation. Analysis of the regulatory elements of the same DARs showed that cis-regulatory regions that change with cessation are highly enriched in pathways related to insulin action, resistance, gluconeogenesis, and metabolism (Supplemental Figure $14 \mathrm{~B})$. Supplemental Figure $14 \mathrm{C}$ shows protein-coding genes $(n=$ $21,955)$ in the form of a heatmap of all proximal peaks $( \pm 1 \mathrm{~kb})$ from the transcription start site (TSS), depicting changes in chromatin accessibility following reversal. Interestingly, SMARCA5 expression, which was shown to be regulated in response to $\mathrm{PM}_{2.5}$ exposure, demonstrated reversibility with exposure cessation (Figure 4D). Two of the most highly regulated genes in the liver were Nr4a1 (Nur77) and Rgs16, which happen to be particularly relevant to the genesis of IR and inflammation $(6,18)$. The impact of cessation on the expression and regulatory elements of the most upregulated (Rgs16) and downregulated (Nr4a1) genes in the liver is depicted in Figure 4, E and F. Epigenomic changes with exposure cessation were concordant with the change in mRNA levels for several circadian genes, providing evidence for the reprogramming of transcription in their promoters such as the Rgs16 (Supplemental Figure 13B) and Bmal1 (Supplemental Figure 15) promoters. The reversible changes in circadian genes seen with cessation of exposure suggest a causality of air pollution exposure in circadian dysregulation.

$\mathrm{PM}_{2.5}$ exposure promoted substantial chromatin remodeling, especially at promoter and enhancer sites that were pliable, with cessation of exposure resulting in a reversal of changes in chromatin accessibility and of expression of transcripts, notably those involved in insulin action, circadian rhythm, and inflammation. An important additional finding was that expression of SMARCA5 (SWI/SNF complex) was regulated in response to $\mathrm{PM}_{2.5}$ exposure and was reversible with exposure cessation. SWI/SNF complexes are a family of polymorphic, ATP-dependent chromatin remodeling complexes that are recruited to cis-regulatory elements such as promoters and enhancers, where they contribute to chromatin accessibility (19). Chromatin remodeling may be particularly important in the context of environmental exposures in which epigenomic changes may be required to buffer and regulate gene expression (17). A growing body of evidence shows that short- and short-term exposure to ambient $\mathrm{PM}_{2.5}$ is associated with altered DNA methylation in specific genes related to inflammation, vascular endothelial dysfunction, and cytokine production and that these effects are implicated in the perturbation of circulating cytokines and fasting blood glucose levels (20-22).

Our study has limitations that must be acknowledged. We did not identify the precise epigenetic regulators that lead to metabolic dysfunction through genome-wide methylation analysis. In addition, histone modifications (e.g., H3K36me3, activation marks; H3K27me3, repression marks; H3K27ac, enhancer marks) and the 3D structure of the chromatin may also be involved in epigenetic reprogramming upon chronic $\mathrm{PM}_{2.5}$ exposure. Our results will require validation in well-designed human studies and, if confirmed, may have important implications for interventions to reduce air pollution levels and exposure.

\section{Methods}

Further information can be found in the Supplemental Methods.

Data availability. Sequencing data from this study have been deposited in the NCBI's Gene Expression Omnibus (GEO) database (GEO GSE145840).

Study approval. All animal procedures and experiments were approved by the IACUC of Case Western Reserve University.

\section{Author contributions}

SR and SB conceptualized and initiated the study and edited and approved the final manuscript. BP wrote an initial draft of the manuscript. $\mathrm{BP}$ and $\mathrm{KDH}$ conducted bioinformatics analysis and created most of the figures. RP, RSG, and YC performed $\mathrm{PM}_{2.5}$ exposure experiments and collected tissue samples. RP conducted phenotype analyses including liver histology studies, microscope imaging, and glucose tolerance tests (GTTs) and insulin tolerance tests (ITTs). JAD conducted data analysis and edited the manuscript. JY assisted with DNA/RNA preparation and performed the qPCR analyses. VV prepared the sequencing libraries and performed OMNI ATAC-Seq. SAK and MKJ reviewed the manuscript and provided critical comments. LD edited the manuscript and figures. All authors helped revise and finalize the manuscript.

\section{Acknowledgments}

The authors wish to thank Adele Snowman, Bindu Paul, and Lie Gao for their assistance with whole brain dissection and Juhyung Woo and Justin Edwards for their help with tissue collection. We thank Alexias Safi and Gregory Crawford at 
Duke University for performing ATAC-Seq using the original protocol for liver samples. We also wish to thank Justin Colacino and Judith S. Opp (University of Michigan) for transcriptome sequencing. This work was supported by grants from the National Institute of Environmental Health Sciences (NIEHS), NIH (U01ES026721, to SB and SR, and R01ES015146 and R01ES019616, to SR).
Address correspondence to: Sanjay Rajagopalan, Cardiovascular Research Institute, Case Western Reserve School of Medicine, 11100 Euclid Avenue, Mailstop Lakeside 5038, Cleveland, Ohio 44106, USA. Email: sxr647@case.edu. Or to: Shyam Biswal, Department of Environmental Health and Engineering, Johns Hopkins University, 615 North Wolfe St., Baltimore, Maryland 21205, USA. Email: sbiswal1@jhu.edu.
1. Landrigan PJ, et al. The Lancet Commission on Pollution and Health. Lancet. 2018;391(10119):462-512.

2. Münzel T, et al. Environmental stressors and cardio-metabolic disease: part II-mechanistic insights. Eur Heart J. 2017;38(8):557-564.

3. Rajagopalan S, Al-Kindi SG, Brook RD. Air Pollution and Cardiovascular Disease: JACC State-of-the-Art Review. J Am Coll Cardiol. 2018;72(17):2054-2070.

4. Sato F, Kohsaka A, Bhawal UK, Muragaki Y. Potential roles of Dec and Bmal1 genes in interconnecting circadian clock and energy metabolism. Int J Mol Sci. 2018;19(3):E781.

5. Agius L. Glucokinase and molecular aspects of liver glycogen metabolism. Biochem J. 2008;414(1):1-18.

6. Chao LC, et al. Insulin resistance and altered systemic glucose metabolism in mice lacking Nur77. Diabetes. 2009;58(12):2788-2796.

7. Shaked I, et al. Transcription factor Nr4a1 couples sympathetic and inflammatory cues in CNSrecruited macrophages to limit neuroinflammation. Nat Immunol. 2015;16(12):1228-1234.

8. Suurväli J, et al. RGS16 restricts the pro-inflammatory response of monocytes. Scand J Immunol. 2015;81(1):23-30.

9. Guo T, Bond ND, Jou W, Gavrilova O, Portas J,
McPherron AC. Myostatin inhibition prevents diabetes and hyperphagia in a mouse model of lipodystrophy. Diabetes. 2012;61(10):2414-2423.

10. Crnko S, Du Pré BC, Sluijter JPG, Van Laake LW. Circadian rhythms and the molecular clock in cardiovascular biology and disease. Nat Rev Cardiol. 2019;16(7):437-447.

11. Bass J, Lazar MA. Circadian time signatures of fitness and disease. Science. 2016;354(6315):994-999.

12. Takahashi JS. Transcriptional architecture of the mammalian circadian clock. Nat Rev Genet. 2017;18(3):164-179.

13. Masri S, Zocchi L, Katada S, Mora E, Sassone-Corsi P. The circadian clock transcriptional complex: metabolic feedback intersects with epigenetic control. Ann N Y Acad Sci. 2012;1264:103-109.

14. Masri S, Sassone-Corsi P. The circadian clock: a framework linking metabolism, epigenetics and neuronal function. Nat Rev Neurosci. 2013;14(1):69-75.

15. Tripodi IJ, Allen MA, Dowell RD. Detecting differential transcription factor activity from ATACSeq data. Molecules. 2018;23(5):E1136.

16. Kulakovskiy IV, et al. HOCOMOCO: towards a complete collection of transcription factor binding models for human and mouse via large-scale ChIP-Seq analysis. Nucleic Acids Res.
2018;46(D1):D252-D259.

17. Padmanabhan K, Billaud M. Desynchronization of circadian clocks in cancer: a metabolic and epigenetic connection. Front Endocrinol (Lausanne). 2017;8:136.

18 . Vivot $\mathrm{K}$, et al. The regulator of $\mathrm{G}$-protein signaling RGS16 promotes insulin secretion and $\beta$-cell proliferation in rodent and human islets. $\mathrm{Mol}$ Metab. 2016;5(10):988-996.

19. Kelso TWR, Porter DK, Amaral ML, Shokhirev MN, Benner C, Hargreaves DC. Chromatin accessibility underlies synthetic lethality of SWI/ SNF subunits in ARID1A-mutant cancers. Elife. 2017;6:e30506.

20. Bind MA, et al. Air pollution and gene-specific methylation in the Normative Aging Study: association, effect modification, and mediation analysis. Epigenetics. 2014;9(3):448-458.

21. Chen R, et al. DNA hypomethylation and its mediation in the effects of fine particulate air pollution on cardiovascular biomarkers: a randomized crossover trial. Environ Int. 2016;94:614-619.

22. Li H, Chen R, Cai J, Cui X, Huang N, Kan H. Short-term exposure to fine particulate air pollution and genome-wide DNA methylation: a randomized, double-blind, crossover trial. Environ Int. 2018;120:130-136. 